\title{
High performance chirped microwave generator for space applications
}

G. Brunetti, Giovanna Marocco, Mario Armenise, Caterina Ciminelli

G. Brunetti, Giovanna Marocco, Mario N. Armenise, Caterina Ciminelli, "High performance chirped microwave generator for space applications," Proc. SPIE 11852, International Conference on Space Optics — ICSO 2020, 118522V (11 June 2021); doi: 10.1117/12.2599455

SPIE Event: International Conference on Space Optics - ICSO 2021, 2021, Online Only 


\section{International Conference on Space Optics-ICSO 2020}

Virtual Conference

30 March-2 April 2021

Edited by Bruno Cugny, Zoran Sodnik, and Nikos Karafolas
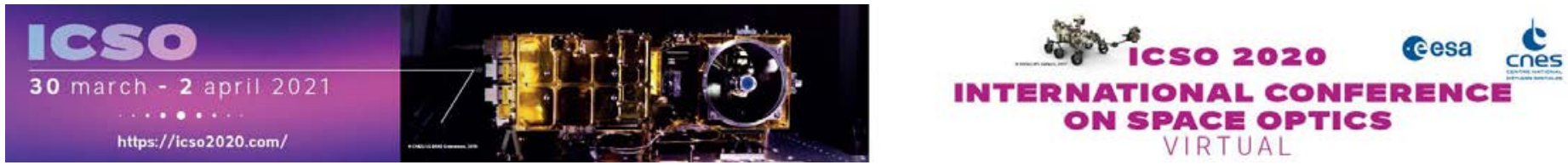

\section{High-performance Chirped Microwave Generator for Space Applications}

\section{Cesa isopmeatians ecnes}




\title{
High-performance Chirped Microwave Generator for Space Applications
}

\author{
Giuseppe Brunetti a, Giovanna Marocco ${ }^{\text {a }}$, Mario N. Armenise ${ }^{\mathrm{a}}$, Caterina Ciminelli*a \\ ${ }^{a}$ Optoelectronics Laboratory, Politecnico di Bari, 70125 Bari (Italy)
}

\begin{abstract}
Photonic generation and transmission of Linearly Chirped Microwave Waveform $(L C M W)$ with a high Time-BandWidth Product (TBWP), of the order of $10^{2}$ or more, is a widely used approach in Synthetic Aperture Radar $(S A R)$ payloads to realize high range resolution, improving the distinction between two or more targets on the same bearing. In standard payload systems, an electronic approach based on voltage-controlled oscillator or digital signal processing, is used as $L C M W$ generator, with both limited $T B W P$ and operating frequency. In this context, a photonic approach plays a crucial role, ensuring low phase noise, high $T B W P$ and operation frequency of the order of GHz, with a significant reduction of mass and size with respect to the electronic counterparts. Here, we propose the design of a Ka-band photonic $L C M W$ generator, based on a frequency-tuneable Opto-Electronic Oscillator $(O E O)$ and a Recirculating Phase Modulation Loop $(R P M L)$, with high spectral purity and high chirp rate in a small footprint. The $O E O$ heart is a one-dimensional photonic crystal ring resonator with an ultra-high $Q$ factor value and an optical delay $>10 \mu$ s. A low-frequency optical signal, with a high chirp rate and $T B W P$ values of the order of $10^{2}-10^{3}$, is generated in the $R P M L$ section.
\end{abstract}

Keywords: Linearly chirped microwave waveform generator, photonic payload, optoelectronic oscillator

\section{INTRODUCTION}

Synthetic Aperture Radar (SAR) has become very popular due to the ability to perform low-cost imaging, unaffected by day-night illumination and weather conditions. $S A R$ allows for high-resolution remote sensing and operates in different $R F$ bands (S-, C-, X-, and Ka-bands), which made it widely used in the field of Earth Observation (EO) [1]. Key building blocks in a $S A R$ are linearly chirped microwave waveform generator, frequency converter, analog-to-digital converter and beamforming network (see Fig. 1). The conventional approach for the Linearly Chirped Microwave Waveform ( $L C M W)$ generation is based on electronic systems as voltage controlled oscillators (VCOs) or digital signal processors $(D S P \mathrm{~s})$. Digital synthesizers or field programmable gate arrays ( $F P G A$ s), together with the analog-to-digital converter ( $A D C$ ), are used to realize the direct digital synthesis of the chirped signal in the baseband, which is then shifted to higher frequencies by means of an up converter (e.g. TerraSAR ${ }^{\mathrm{TM}}$ [2]). This approach shows a Time-BandWidth Product (TBWP) of around 1000 with a center frequency and bandwidth limited to a few gigahertz. The choice of electronic components constitutes a limit to the speed and bandwidth of the system and does not meet the necessary requirements for new generation $S A R$ payloads. Recently, schemes without up-converters have been presented, but the limit due to the presence of electronic components stays in place. The central frequency and bandwidth of the chirped electronic generator are limited to a few gigahertz [3].

Integrated photonics can overcome these limitations. Microwave photonics technology has great potential in realizing SAR payload systems, with wide detection range and good resolution. The use of photonic components allows not only to reduce size and mass of the payload, but also to ensure a more efficient control of the deployment of the antenna in orbit, and to implement subsystems traditionally made electronically, such as beamforming networks, high-precision sensors, analog RF front-ends, analog-to-digital converters, [4].

Low phase noise and higher $T B W P$ are the further advantages of the photonic approach alongside the compatibility of this technology with the new generation operating bandwidths, i.e. the Ka-band (26.5 - $40 \mathrm{GHz})$, of interest to several space agencies. Future space $E O$ missions require operating bands much larger than the X-band (e.g. CosmoSkyMed in X-band [5]) or S-band (e.g NovaSAR-1 [6]) due to the need to guarantee higher resolution and speed.

*caterina.ciminelli@poliba.it 


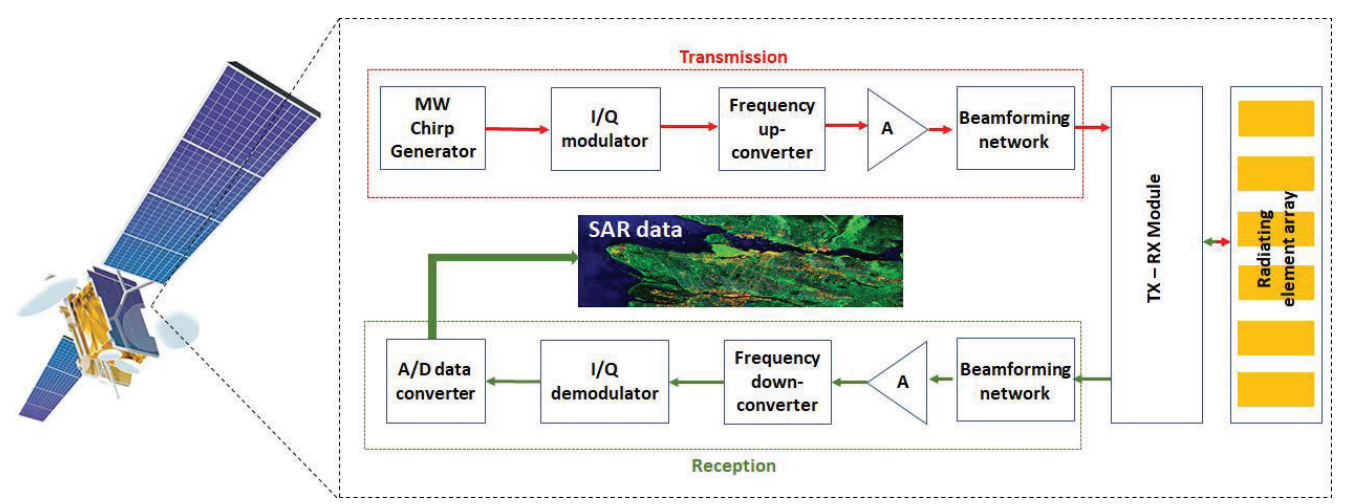

Figure 1: Design scheme of a SAR payload. MW: microwave; A/D: analog-to-digital; Tx: transmission; Rx: reception (Inset image credit: RADARSAT-2 Data and Products (C) MacDonald Dettwiler and Associates Ltd (2009)).

Several configurations of photonic $L C M W$ generators have been proposed in literature [7]. The most common architectures are based on self-heterodyne technique, spectral shaping and frequency- to-time mapping technique (SS-FTT), optically injected semiconductor laser and the combination of a frequency-tunable $O E O$ and a recirculating phase modulation loop $(R P M L)$.

The first approach is based on optical heterodyning technology by beating one sweeping optical signal with a continuous wave light. A TBWP of 4200, with a long duration of $1 \mu$ s and a bandwidth of $4.2 \mathrm{GHz}$, have been reported [8]. Despite of these good performance, this method suffers from a large phase noise due to non-coherent light sources.

The SS-FTT method is based on shaping an ultrashort optical pulse by an optical spectral shaper containing a Sagnac loop, a Fourier transform pulse shaper and fiber Bragg gratings [9]. A dispersive medium executes the conversion to the time domain and produces the chirped pulse. The easy implementation makes this technique interesting, but the method presents $T B W P$ in the order of tens at central frequency $>20 \mathrm{GHz}$.

Period-one dynamics of an optically injected modulated laser [10] or dual-mode state of a single monolithically integrated amplified feedback laser [11] are used to develop injected laser-based chirp generator technique. Many discrete photonic components are required for the former approach, making the configuration bulky. An ultra-wide TBWP $\left(\right.$ e.g. $\left.5.159 \times 10^{5}\right)$ with a bandwidth larger than $5 \mathrm{GHz}$ can be implemented with the latter approach, which presents a more compact solution. An attractive approach has been proposed by [12], where a TBWP of the order of tens has been achieved through the beating the output of a tunable $O E O$ and of a $R P M L$, controlled by a parabolic signal. Although performance are interesting, the architecture is bulky due to the presence of discrete photonic components.

In this paper, we propose the design of a system in package Ka-band photonic $L C M W$ generator, based on a frequencytunable $O E O$ and a $R P M L$, with high $T B W P$ in a small footprint. The $O E O$ core is a one-dimensional photonic crystal ring resonator with an ultra-high $Q$ factor value and an optical delay $>10 \mu \mathrm{s}$, that ensures a phase noise@10 KHz from the carrier less than $-120 \mathrm{dBc} / \mathrm{Hz}$. A low-frequency optical signal, with a high chirp rate and $T B W P$ of the order of $10^{2}-10^{3}$, is generated in the $R P M L$ section by using multiple phase modulations and an suitable waveform as the driven voltage of the phase modulator. The Ka-band signal with a power of about $-10 \mathrm{dBm}$ and a $T B W P$ in the range $500-1000$ is generated through the beating of the output beams of the $O E O$ and $R P M L$ at a high-speed photodiode.

\section{SYSTEM DESCRIPTION}

The proposed architecture is shown in Fig. 2 and consists of two sections: a tunable $O E O$ and a $R P M L$, based on the system proposed in [12]. A continuous-wave light signal is generated by a tunable external cavity laser, that consists of a InPbased gain chip and a planar lightwave circuit including a Bragg grating, with an output power of $20 \mathrm{~mW}$, a linewidth $\leq 2$ $\mathrm{kHz}$ and a Relative Intensity Noise $(R I N)$ of $-140 \mathrm{~dB} / \mathrm{Hz}$ [13]. The continuous signal is routed in two branches by using a $3 \mathrm{~dB}$ silicon power splitter [14]. One replica feeds an InP-based Mach-Zehnder modulator $(M Z M)$ [15], electrically driven by square wave voltage, that generates a chirp free optical pulse. The other replica enters a phase modulator $(P M)[16]$ in the $O E O$ to obtain a frequency-tunable microwave signal in the Ka-band. The output of the $M Z M$ is then sent to the $R M P L$ section, where multiple phase modulations at the $P M$, driven by a splitting parabolic waveform, increases the chirp rate. When the signal reaches the desired chirp rate, it is routed out by using a silicon MZI switch, driven by a square wave voltage [17]. 


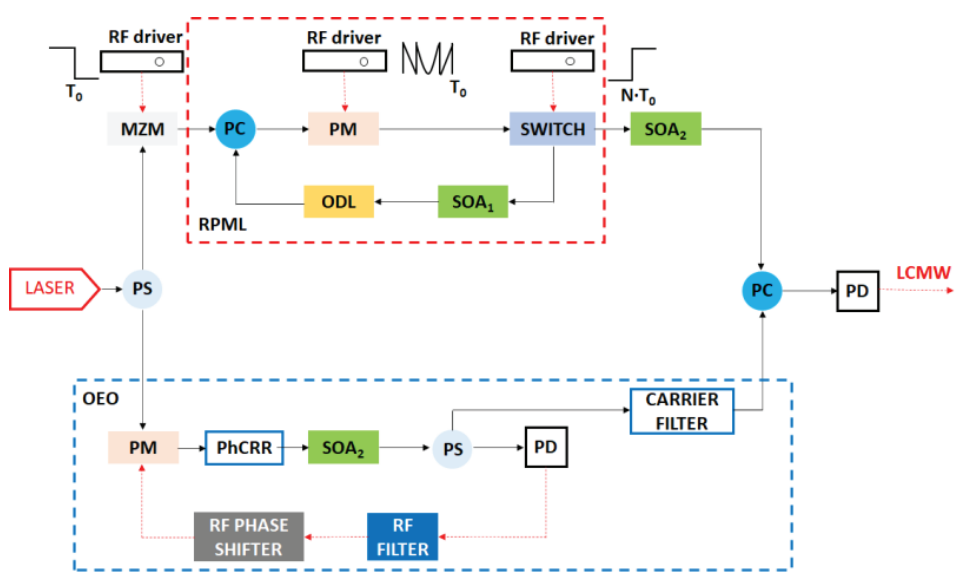

Figure 2. Configuration of the proposed LCMW generator ( $P S$ : power splitter, MZM: Mach-Zehnder interferometer Modulator, $P C$ : power combiner, $P M$ : phase modulator, SOA: Semiconductor Optical Amplifier, $O D L$ : optical delay line, PhCRR: Photonic Crystal Ring Resonator, $P D$ : photodiode).

The $L C M W$ is generated by beating the output of the $O E O$ and $R P M L$ sections at the photodiode $(P D)$, that operates at $1.55 \mu \mathrm{m}$ with a responsivity of $0.7 \mathrm{~A} / \mathrm{W}$, a dark current of $150 \mathrm{nA}$, a total resistance of $100 \Omega$ and a bandwidth $>60 \mathrm{GHz}$ [18].

The generator can be fabricated by a very innovative technological platform [19], which allows the bonding-enabled heterogeneous integration of Si-derived photonic components with InP-based high-performance optoelectronic devices, while, the RF components of the $O E O$ section can be integrated on a $M M I C$, that could be packaged with the optoelectronic section, so, creating a system-in-package.

In the following, the e.m. behavior of the proposed system is mathematically described. The lightwave $E_{l}(t)$ at the output of the RMPL section can be expressed as:

$$
E_{1}(t)=E_{01} \cdot s(t) \cdot \exp \left[j\left(2 \pi f_{0} t+N \frac{\pi V}{V_{\pi}} c(t)\right)\right]
$$

where $E_{01}$ is the amplitude of $E_{1}, f_{0}$ is the operating frequency of the light source $(\approx 193 \mathrm{THz}), s(t)$ is driven voltage of the $M Z M$ (see Eq. 2), $c(t)$ is the splitted parabolic waveform that drives the $P M$ in the RPML (see Eq. 3), $N$ is the number of the round-trips in the RPML section, $V$ is the peak-to-peak voltage of the parabolic waveform [12] and $V_{\pi}$ is the half-wave voltage of the PM.

$$
\begin{gathered}
\mathrm{s}(t)=\left\{\begin{array}{cc}
1 & t \leq T_{0} \\
0 & \text { else }
\end{array}\right. \\
c(t)=\frac{K \cdot M}{2}\left\{\begin{array}{cc}
\left(t-T_{0} / 2\right)^{2}-\left(t_{n}(1)-T_{0} / 2\right)^{2} & t_{n}(0) \leq t \leq t_{n}(1) \\
\left(t-T_{0} / 2\right)^{2}-\left(t_{n}(2)-T_{0} / 2\right)^{2} & t_{n}(1) \leq t \leq t_{n}(2) \\
\vdots & \vdots \\
\left(t-T_{0} / 2\right)^{2}-\left(t_{n}(M / 2+1)-T_{0} / 2\right)^{2} & t_{n}(M / 2) \leq t \leq t_{n}(M / 2+1) \\
\left(t-T_{0} / 2\right)^{2}-\left(t_{p}(0)-T_{0} / 2\right)^{2} & t_{p}(0) \leq t \leq t_{p}(1) \\
\left(t-T_{0} / 2\right)^{2}-\left(t_{p}(1)-T_{0} / 2\right)^{2} & t_{p}(1) \leq t \leq t_{p}(1) \\
\vdots & \vdots \\
\left(t-T_{0} / 2\right)^{2}-\left(t_{p}(M / 2)-T_{0} / 2\right)^{2} & t_{p}(M / 2) \leq t \leq t_{p}(M / 2+1)
\end{array}\right.
\end{gathered}
$$

where $M$ is the number of splitting, $T_{0}$ is temporal duration of the signal, $K=\frac{4}{T_{0}^{2}}, t_{n}(i)=\frac{T_{0}}{2}-\sqrt{\frac{M-i}{K \cdot M}}$, and $t_{p}(i)=\frac{T_{0}}{2}+\sqrt{\frac{i}{K^{\cdot} \cdot M}}$. The lightwave $E_{2}(t)$ at the output of the $O E O$ section could be expressed as: 


$$
E_{2}(t)=E_{02} \cdot \exp \left[j\left(2 \pi\left(f_{0}-f_{\text {osc }}\right) t\right)\right]
$$

where $E_{02}$ is the amplitude of $E_{2}, f_{\text {osc }}$ is the oscillating frequency $(\approx 193.04 \mathrm{THz})$.

The $L C M W$ is generated by beating the two lightwaves at the $P D$. In particular, by neglecting the DC component, the $P D$ output $i(t)$ can be expressed as:

$$
i(t) \propto\left[E_{I}(\mathrm{t})+E_{2}(t)\right]^{2}=\left\{\begin{array}{cc}
\cos \left[2 \pi f_{\text {osc }} t+\frac{N \pi V}{V_{\pi}} \frac{M K}{2}\left(\left(t-\frac{T_{0}}{2}\right)^{2}-a_{i}\right)\right] & \begin{array}{c}
t \leq T_{0} \\
0
\end{array} \\
\text { else }
\end{array}\right.
$$

where the parameter $a_{i}$ is extracted by Eq. (3) $\left(a_{i}=\left(t_{n}(i)-T_{0} / 2\right)^{2}\right.$ for $t_{n}(0) \leq t \leq t_{n}(M / 2+1), a_{i}=\left(t_{p}(i)-T_{0} / 2\right)^{2}$ for $t_{p}(0) \leq t \leq t_{p}(M / 2+1)$.

The $T B W P$ has been calculated by calculating the instantaneous frequencies $\left(f_{\text {ist, } m a x}, f_{i s, \text { min }}\right)$ from Eq. (5):

$$
T B W P=T_{0}\left|f_{i s t, \max }-f_{i s t, \text { min }}\right|=\frac{2 \cdot M \cdot N \cdot V}{V_{\pi}}
$$

By assuming the signal duration $T_{0}=15 \mathrm{~ns}$ and a high speed travelling wave InGaAsP/InP phase modulator, with a bandwidth up to $40 \mathrm{GHz}, V_{\pi}=1.8 \mathrm{~V}$, and insertion loss (IL) equal to $3 \mathrm{~dB}$ [16] as $P M$ in the $R P M L$, the $T B W P$ behavior by varying $V, M$, and $N$ has been illustrated in Fig. 3 .

The electric response of the $L C M W$ generator has been calculated by using a system level simulator for realistic modelling of complex system, including optical and optoelectronic components [20].

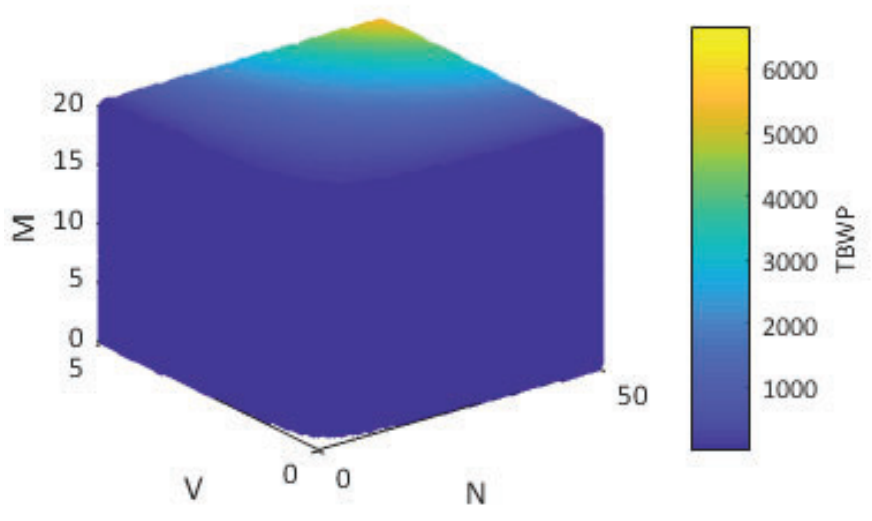

Figure 3. $T B W P$ vs. $N, V$, and $M$, according to Eq. (6).

\subsection{Recirculating Phase Modulation Loop (RPML)}

The first module in the generator (Fig. 2) is the RPML. It consists of a silicon power combiner $(P C)$ [14], a InGaAsP/InPbased $P M$ [16], a 2x1 silicon optical switch [17], a Semiconductor Optical Amplifier (SOA) (SOA $A_{1}$ in Fig. 2) [21] and a $\mathrm{Si}_{3} \mathrm{~N}_{4}$ optical delay line.

A chirp free signal is generated by a $M Z M$ driven by the signal $s(t)$. The signal is $N$-times phase modulated by the $P M$, driven by a splitted parabolic signal $c(t)$ with peak-to-peak amplitude $V$, until the switch is "off" $\left(\approx N \cdot T_{0}\right)$. When the switch is in the state "on", the chirped signal is routed out of RMPL and it is amplified by a $S O A$ ( $\mathrm{SOA}_{2}$ in Fig. 2) [22].

For the performance estimation, a silicon MZI 2x1 optical switch (SWITCH in Fig. 2) has been assumed, having a crosstalk of $30 \mathrm{~dB}$, insertion loss of $0.05 \mathrm{~dB}$ and rise time and fall time equal to $3.2 \mathrm{~ns}$ and $2.5 \mathrm{~ns}$, respectively [17]. The $M Z M$ is a $\mathrm{InP} / \mathrm{Si}$ - based $M Z I$ with an extinction ratio of $52 \mathrm{~dB}[15]$.

The main goal of the optical delay line $(O D L)$ is to synchronize the optical signal circulating in the RPML, aiming at achieving a delay of $15 \mathrm{~ns}$ to avoid the self-overlapping of the signal at the $P M$ and to properly extract the signal when the switch is "on". To this aim, a custom optical delay line has been designed. It consists of a Fermat spiral, based on a $\mathrm{Si}_{3} \mathrm{~N}_{4}$ $100 \mathrm{~nm} \times 2.8 \mu \mathrm{m}$ waveguide [23], with a minimum radius of $4 \mathrm{~mm}$ [24]. The spiral has a footprint of $3.8 \mathrm{~cm} \times 3.8 \mathrm{~cm}$ and a time delay of $14 \mathrm{~ns}$ with a propagation loss of $9.8 \mathrm{~dB}$, calculated for the TE mode. To compensate the propagation losses 
of the $P M$, SWITCH and $O D L$ in Fig. 2, a $S O A$ with a gain of $13 \mathrm{~dB}$ and a noise figure of $4 \mathrm{~dB}$ has been included in the feedback loop [21].

To properly design the shape of signal $c(t)$, by taking into account the electronic constraints, a maximum value $M$ equal to 16 has been considered (see Fig. 3(a)) with $V=1.5 \mathrm{~V}$. The output of the $R P M L$ by varying $N$ is shown in Fig. 3(b). A $T B W P$ of 532 and 1061 has been calculated for $N=20$ and 40, respectively, that fit with the predicted values by using Eq. (6) $(T B W P=533$ for $N=20, T B W P=1066$ for $N=40)$.

According to the "law of the conservation of the power", the bandwidth increase leads to a significant decrease of the optical power (see Fig. 3(b)), with an exponential trend. For $N=40$, a power loss of $26 \mathrm{~dB}$ with respect the carrier has been calculated. Therefore, a $\mathrm{SOA}\left(\mathrm{SOA}_{2}\right.$ in Fig. 2) with gain of $25 \mathrm{~dB}$ and a noise figure of $8 \mathrm{~dB}$ [22], has been considered at the end of the RPML to compensate the aforementioned losses.

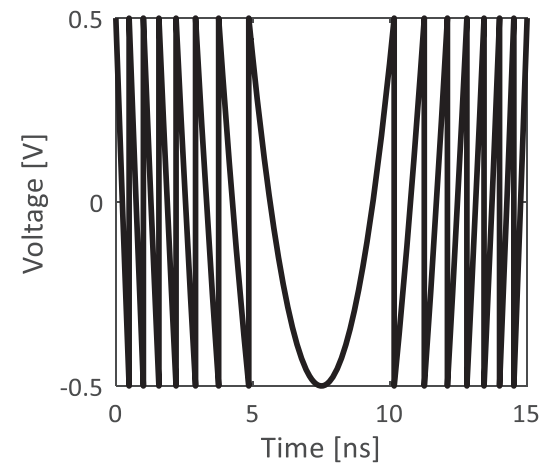

(a)

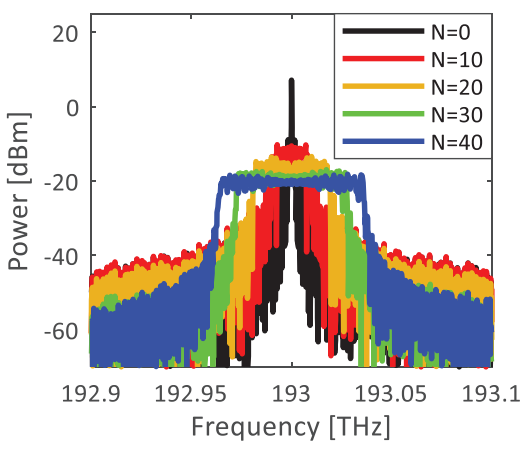

(b)

Figure 4. (a) $c(\mathrm{t})$ with $M=16$. (b) $R P M L$ spectra by varying $N(M=16, V=1.5 \mathrm{~V})$.

\subsection{Optoelectronic Oscillator $(O E O)$}

The second building block in Fig. 2 is the $O E O$. The lightwave from the power splitter is phase modulated by the $P M[16]$. The optical beam at the $P M$ output passes through the sensitive element $(P h C R R)$, that acts as optical filter and time delay element $[24,25]$, is amplified by the $\mathrm{SOA}_{2}$ [11], and, then, $\mathrm{O} / \mathrm{E}$ transduced by a photodiode. The photodiode output is filtered, phase shifted and then fed back to the modulator. The output beam is routed out by a $P S$ [14] after the $S O A_{2}$. The self-sustained oscillation is due to the positive feedback loop and it is generated when the $\mathrm{SOA}_{2}$ compensates the optical losses into the loop [26, 27].

The oscillator key building block is a $\mathrm{Si}_{3} \mathrm{~N}_{4}$ ring resonator including a $1 \mathrm{D}$ photonic crystal (PhC) in the resonant path $(P h C R R)$ (see Fig. 5(a)). The periodic structure consists of a top grating in an ultra-low-loss $\mathrm{Si}_{3} \mathrm{~N}_{4}$ strip waveguide [23], obtained by modulating the thickness of the waveguide with a width $w=2.8 \mu \mathrm{m}\left(t_{H}=100 \mathrm{~nm}\right.$ and $\left.t_{L}=60 \mathrm{~nm}\right)$. The Si ${ }_{3} \mathrm{~N}_{4}$ bus waveguide has a thickness $t$ of $100 \mathrm{~nm}$ and a width $w$ of $2.8 \mu \mathrm{m}$, to ensure a single mode operation, placed on top of a thermal $\mathrm{SiO}_{2} 15 \mu \mathrm{m}$ thick. A period $\Lambda$ of $526 \mathrm{~nm}$ has been designed for the grating in order to operate at about $1550 \mathrm{~nm}$. A propagation loss of $3.5 \mathrm{~dB} / \mathrm{m}$ has been assumed for the bus waveguide [23] while, for the grating, a propagation loss value one order of magnitude higher has been considered to take into account additional losses due to the grating fabrication. A radius $R=2 \mathrm{~mm}$ has been considered with a value of gap $=4.28 \mu \mathrm{m}$. The e.m. behavior has been calculated by using the mathematical model reported in $[24,28]$. The spectral response shows a photonic bandgap centered at Bragg wavelength and band edge resonances with a variable free spectral range. The slow-light effect ensures ultra-high values of the $Q$-factor. The first band-edge resonance shows a $Q$-factor of $5.64 \times 10^{9}$ with an extinction ratio of about $10 \mathrm{~dB}$ (see Fig. 5(b)).

The laser frequency $f_{0}$ has to set in accordance with the resonance frequency of the optical cavity $f_{R}$, aiming at achieving a filtering at $f_{0}+40 \mathrm{GHz}$. For the stabilization of the laser frequency, the Pound-Drever-Hall technique can be used [29]. For simplicity, $f_{0}=193 \mathrm{THz}$ and the frequency resonance $f_{R}$ of the PhCRR at $193.04 \mathrm{THz}$ have been considered.

According to the $O E O$ phase noise model based on the well-known Leeson's approach developed in [30], a phase noise at $10 \mathrm{kHz}$ from the carrier $<-120 \mathrm{dBc} / \mathrm{Hz}$ has been predicted, that results comparable with the state-of-the-art [31], within a very small footprint. 


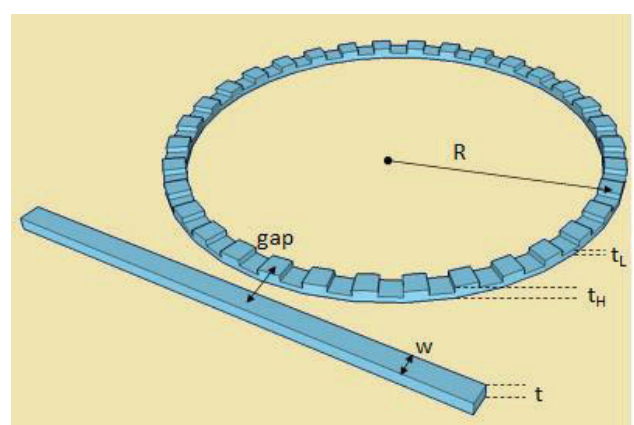

$\mathrm{Si}_{3} \mathrm{~N}_{4}$

$\mathrm{SiO}_{2}$

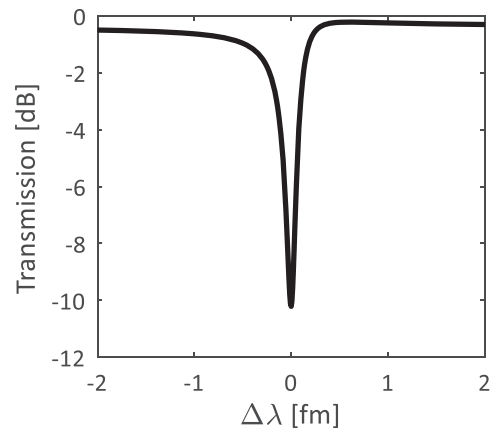

(a)

(b)

Figure 5. (a) Photonic Crystal Ring Resonator $(P h C R R)$, (b) Transmission spectrum of PhCRR with $R=2$ mm and gap $=$ $4.28 \mu \mathrm{m}\left(\Delta \lambda\right.$ : wavelength shift with respect to $\left.f_{R}\right)$.

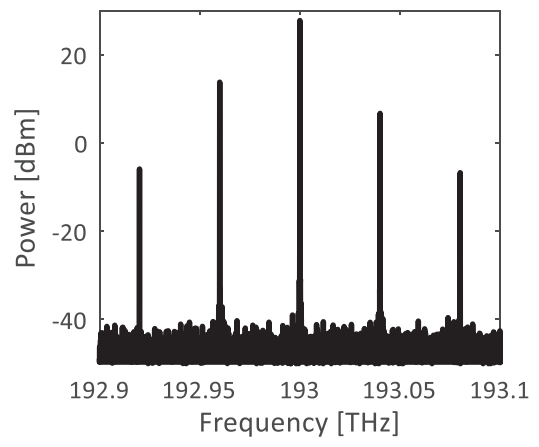

Figure 6. $R P M L$ output spectrum.

The output of the $O E O$ section is reported in Fig. 6, where, besides the carrier, spectral components at multiples and submultiples of $40 \mathrm{GHz}$ appear. In order to linearly chirped modulating only the spectral component at $40 \mathrm{GHz}$, the suppression of the carrier and $40 \mathrm{GHz}$ submultiples/multiples is needed. Therefore, the modulation index of 0.8 [32] and the use of a carrier filter with an ultra-high extinction ratio represents a good compromise. The filter at the output of the $O E O$ consists of a silicon $M Z I$, with one branch coupled to a ring resonator and the other to three serially coupled ring resonators, with an extinction ratio $=150.55 \mathrm{~dB}$ and a bandwidth $=0.243 \mathrm{~nm}$, within a footprint of $60 \mu \mathrm{m} \times 160 \mu \mathrm{m}$ [33]

\section{LCMW GENERATOR PERFORMANCE}

The $L C M W$ results by beating the outputs of the $R P M L$ and $O E O$ at the $P D$. The electrical responses at the output for $N=$ 20 and $N=40$ are reported in Fig. 7(a)-(b), respectively. $N=20$ ensures a $T B W P$ of 540 at $40 \mathrm{GHz}$ with a mean output power of $-12 \mathrm{dBm}$ and a $R F$ spurious suppression ratio (RFSSR) of $10 \mathrm{~dB}$, calculated as the worst difference between the optical power of the $L C M W$ and the spectral components outside the bandwidth. For $N=40$ a TBWP of 1076 at $40 \mathrm{GHz}$, an output power of about $-15 \mathrm{dBm}$ and a $R F S R R$ of $7 \mathrm{~dB}$ have been calculated. Since $N=40$ leads a bandwidth of about $66 \mathrm{GHz}$, the lower value of $R F S S R$ with respect $N=20$ (bandwidth equal to $33 \mathrm{GHz}$ ) is caused by a partial overlapping of the $L C M W$ signals centered at $0 \mathrm{GHz}$ and $80 \mathrm{GHz}$. 


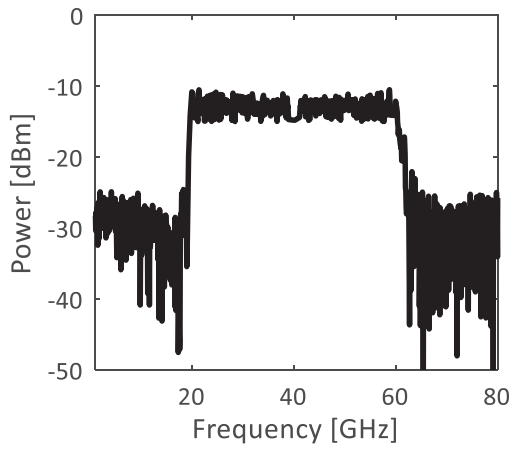

(a)

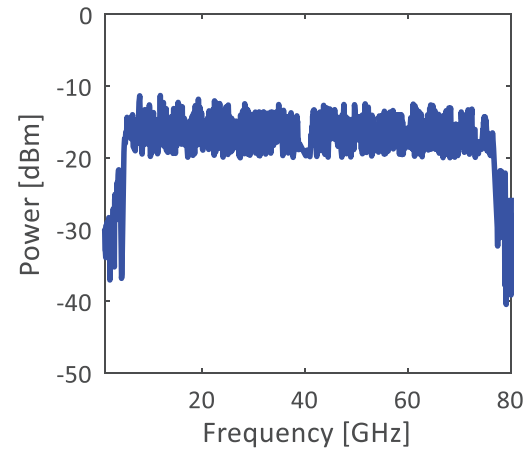

(b)

Figure 7. $L C M W$ for $N=20$ (a) and $N=40(\mathrm{~b})$.

\section{CONCLUSIONS}

The design of a $K a$-band $L C M W$ generator, with a TBWP larger than 500 and 1000 and an output power larger than -15 $\mathrm{dBm}$, has been reported in the paper. The proposed device consists of a $R P M L$ section, whose key element is a $P M$ driven by an engineered splitted waveform able to provide a large $T B W P$, and an $O E O$ section, whose key building block is a $P h C R R$ with a $Q$-factor larger than $10^{9}$ that ensures a phase noise $<-120 \mathrm{dBc} / \mathrm{Hz}$. To guarantee the compactness and the scalability of the device, it can be fabricated by innovative technological platform allowing the hybrid integration of ultralow loss passive devices with active ones. The calculated performance is suitable for $K a$-band $S A R$ system with a high range resolution.

\section{FUNDINGS}

The work has been supported by Ministry of Research and University in the framework of Close to the Earth project (no. ARS01_00141).

\section{REFERENCES}

[1] Armenise, M.N., Dell'Olio, F., Ciminelli, C., Galeone, C., and Brunetti, G., "Microphotonics-based architectures of mini-SAR payloads," Proc. $21^{\text {st }}$ International Conference on Transparent Optical Networks (ICTON), 1-4, (2019).

[2] Pilz, D., and Feldle, P., "RF-payload of TerraSAR-X," Proc. German Microwave. Conference (GeMiC), 140143, (2005).

[3] Srivastava, S. and Hobden, P., "5Ghz Chirp Signal Generator for Broadband FMCW Radar Applications," Proc. IEEE International Symposium on Smart Electronic Systems (iSES), 152-155, (2018).

[4] Dell’Olio, F., Brunetti, G., Conteduca, D., Giovinazzi, N., Sasanelli, N., Ciminelli, C., and Armenise, M.N., "Integrated microwave photonics: Overview and promising space applications," Applications in Electronics Pervading Industry, Environment and Society, Springer, 512 (2019).

[5] Moreira, A., "Synthetic aperture radar (SAR): Principles and applications," Proc. $4^{\text {th }}$ Advanced Training Course in Land Remote Sensing, 1-62, (2013).

[6] Cohen, M., Larkins, A., Semedo, P. L., and Burbidge, G., "NovaSAR-S low cost spaceborne SAR payload design, development and deployment of a new benchmark in spaceborne radar," Proc. IEEE Radar Conference (RadarConf), 0903-0907, (2017).

[7] Wang, X., Ma, J., Zhang, Q., and Xin, X. "Generation of linear frequency-modulated signals with improved timebandwidth product based on an optical frequency comb," Applied Optics, 58(12), 3222-3228, (2019).

[8] Coutinho, O.L., Zhang, J., and Yao, J., "Photonic generation of a linearly chirped microwave waveform with a large time-bandwidth product based on self-heterodyne technique," International Topical Meeting on Microwave Photonics (MWP), 1-4, (2015). 
[9] Wang, C., and Yao, J., "Chirped Microwave Pulse Generation Based on Optical Spectral Shaping and Wavelength-to-Time Mapping Using a Sagnac Loop Mirror Incorporating a Chirped Fiber Bragg Grating,” IEEE J. Lightwave Technol., 27(16), pp. 3336-3341, (2009).

[10]Zhou, P., Zhang, F., Guo, Q., and Pan, S., "Linearly chirped microwave waveform generation with large timebandwidth product by optically injected semiconductor laser," Optics Express, 24(16), 18460-18467, (2016).

[11]Jin Li, J., Pu, T., Zheng, J., Zhang, Y., Shi, Y., Zhu, H., Li, Y., Zhang, X., Zhao, G., Zhou, Y., and Chen, X., "Photonic generation of linearly chirped microwave waveforms using a monolithic integrated three-section laser", Optics Express, 26(8), 9676-9685, (2018).

[12]Li, W., and Yao, J., "Generation of linearly chirped microwave waveform with an increased time-bandwidth product based on a tunable optoelectronic oscillator and a recirculating phase modulation loop," J. Lightwave Technology, 32(20), 3573-3579 (2014).

[13] Datasheet of the RIO PLANEXTM External Cavity Laser, http://www.rio-lasers.com.

[14] Sheng, Z., Wang, Z., Qiu, C., Li, L., Pang, A., Wu, A., Wang, X., Zou S., and Gan, F., “A compact and low-loss MMI coupler fabricated with CMOS technology," IEEE Photonics Journal, 4(6), 2272-2277, (2012).

[15]Zhang, C., Morton, P.A., Khurgin, J.B., Peters, J.D., and Bowers, J.E., "Highly linear heterogeneous-integrated Mach-Zehnder interferometer modulators on Si," Optics Express, 24(17), 19040-19047, (2016).

[16]Zhang, L., Sinsky, J., Van Thourhout, D., Sauer, N., Stulz, L., Adamiecki, A., and Chandrasekhar, S., "Lowvoltage high-speed travelling wave InGaAsP-InP phase modulator", IEEE Photonics Technology Letters, 16(8), 1831-1833, (2004)

[17]Lu, L., Zhao, S., Zhou, L., Li, D., Li, Z., Wang, M., Li X., and Chen, J., “16× 16 non-blocking silicon optical switch based on electro-optic Mach-Zehnder interferometers," Optics Express, 24(9), 9295-9307, (2016).

[18] Shen, L., Jiao, Y., Yao, W., Cao, Z., van Engelen, J. P., Roelkens, G., Smit, M.K., and van der Tol, J.J.G.M., "High-bandwidth uni-traveling carrier waveguide photodetector on an InP-membrane-on-silicon platform," Optics Express, 24(8), 8290-8301, (2016).

[19]Piels, M., Bauters, J.F., Davenport, M.L, Heck, M.J.R., and Bowers, J.E., "Low-Loss Silicon Nitride AWG Demultiplexer Heterogeneously Integrated With Hybrid III-V/Silicon Photodetectors,” J. Lightwave Technol. 32(4), 817-823, (2014).

[20] Optisystem by Optiwave version 16.

[21] Thorlabs SOA1013S - C-Band Semiconductor Optical Amplifier, https://www.thorlabs.com $\backslash$ thorproduct.cfm?partnumber=SOA1013S

[22] Connelly, M.J. "Wideband semiconductor optical amplifier steady-state numerical model," IEEE Journal of Quantum Electronics, 37(3), 439-447, (2001).

[23] Bauters, J.F., Heck, M.J., John, D., Dai, D., Tien, M.C., Barton, J.S., Leinse, A., Heideman, R.G. Blumenthal, D.J. and Bowers, J.E., "Ultra-low-loss high-aspect-ratio Si3N4 waveguides,” Optics Express, 19(4), 3163-3174, (2011).

[24] Brunetti, G., Dell'Olio, F., Conteduca, D., Armenise, M. N., and Ciminelli, C., "Comprehensive mathematical modelling of ultra-high Q grating-assisted ring resonators," Journal of Optics, 22(3), 035802, (2020).

[25] Ciminelli, C., Campanella, C.E., and Armenise, M.N., U.S. Patent No. 9,863,771. Washington, DC: U.S. Patent and Trademark Office, (2018).

[26] Tang, J., Hao, T., Li, W., Domenech, D., Baños, R., Muñoz, P., Zhu, N., Capmany, J., and Li, M., 'Integrated optoelectronic oscillator," Optics Express, 26(9), 12257-12265, (2018).

[27] Ilchenko, V.S., Byrd, J., Savchenkov, A.A., Matsko, A.B., Seidel, D., and Maleki, L., "Miniature oscillators based on optical whispering gallery mode resonators," Proc. IEEE International Frequency Control Symposium, 305 308, (2008).

[28] Ciminelli, C., Innone, F., Brunetti, G., Conteduca, D., Dell'Olio, F., Tatoli, T., and Armenise, M.N., "Rigorous model for the design of ultra-high Q-factor resonant cavities," Proc. $18^{\text {th }}$ International Conference on Transparent Optical Networks (ICTON), 1-4, (2016).

[29] Saleh, K., Lin, G., and Chembo, Y.K., "Effect of laser coupling and active stabilization on the phase noise performance of optoelectronic microwave oscillators based on whispering-gallery-mode resonators," IEEE Photonics Journal, 7(1), 1-11, (2014).

[30] Bouchier, A., Saleh, K., Merrer, P.H., Llopis, O., and Cibiel, G., "Theoretical and experimental study of the phase noise of opto-electronic oscillators based on high quality factor optical resonators," Proc. IEEE International Frequency Control Symposium, 544-548, (2010).

[31] Preliminary product brief of the Micro-Opto-Electronic Oscillator (uOEO), http://www.oewaves.com/ 
[32] Saleh, K. "High spectral purity microwave sources based on optical resonators," Université Paul Sabatier Toulouse III, PhD Thesis, (2012).

[33] Brunetti, G., Sasanelli, N., Armenise, M. N., and Ciminelli, C., "High performance and tunable optical pumprejection filter for quantum photonic systems," Optics \& Laser Technology, 139, 106978, (2021). 